\title{
INVESTIGATION OF ACOUSTIC AND ELECTRICAL PROPERTIES OF A LIP0, METAPHOSPHATE GLASS
}

The acoustic attenuation spectroscopy in connection with conductivity and dielectric relaxation spectroscopy can be useful techniques for the study of relaxation processes and transport mechanisms in Lit ion conducting glasses. A theoretical model and mathematical fit of acoustic and conductivity measurements are used for characterization of the ionic hopping motion, relaxation processes and transport mechanisms connected with the mobility of conducting $\mathrm{Li}^{+}$ions. Results from acoustic measurements are compared with results obtained from electrical conductivity measurements.

Keywords: Acoustic spectroscopy, conductivity spectroscopy, dielectric spectroscopy, Li phosphate glasses, relaxation and transport processes.

\section{Introduction}

The research of lithium conducting glasses has recently seen an increase due to their potential application mainly as solid-state amorphous electrolytes in secondary batteries and the advantages they may offer [1]. In a secondary lithium ion battery, Li metal can be used as the anode material allowing higher energy and power densities than when graphitized anodes are used as anode materials, and one of the most important requirements of the electrolyte material in such a system is to have a high ionic conductivity and being compatible with the electrode materials. The use of solid electrolytes in rechargeable batteries can provide numerous advantages such as the possibility of miniaturization through thin-film production, longer active life, high reliability, thermal, chemical and mechanical stability. Most of the works have been focused on the ionic conductivity of LiPON thinfilms, which is generally given around $2 \times 10^{-6} \mathrm{~S} / \mathrm{cm}$ at $25^{\circ} \mathrm{C}$ [2] and average activation energy of $\mathrm{E}_{\mathrm{a}}=(0.55 \pm 0.02) \mathrm{eV}$ [3] LiPON thin-film electrolytes have been recently studied and their properties and structure compared to those found in lithium phosphate glasses and their oxynitride counterparts [4 and 5]. The structure of a $\mathrm{LiPO}_{3}$ metaphosphate glass is known to be built of long chains or rings of $\mathrm{PO}_{4}$ tetrahedra [6]. Two of the oxygens in each $\mathrm{PO}_{4}$ are bridging oxygens that link together neighbouring groups while the other two are non-bridging oxygens bonded to the modifier cations $\mathrm{Li}^{+}$. On the other hand, the structure of an oxynitride glass will be formed by $\mathrm{PO}_{4}$ and also by new groups,
$\mathrm{PO}_{3} \mathrm{~N}$ and $\mathrm{PO}_{2} \mathrm{~N}_{2}$ tetrahedra, having nitrogen in two different bonding states, as dicoordinated $-\mathrm{N}=\left(\mathrm{N}_{\mathrm{d}}\right)$ or tricoordinated $-\mathrm{N}<$ $\left(\mathrm{N}_{\mathrm{t}}\right)$ nitrogen species. The study of the relaxation processes taking place in these glasses can be important for the future development of all solid-state electrolytes.

The acoustic attenuation measurements seem to be a useful technique for investigation of transport mechanisms in conducting glasses and compared to the electrical one it has even some advantages as high sensitivity, the absence of contact phenomena and several others [7]. Acoustical measurements made over a wide range of frequencies and temperatures can characterize different relaxation processes according to corresponding transport mechanisms due to the strong acousto-ionic interaction and it can discover also different kinds of sites and/or ionic hopping motions associated with the charge mobility [8]. The dielectric spectroscopy in principle derived from the same experimental data as electrical conductivity spectroscopy (i.e., real and imaginary components of the sample impedance) is another powerful technique for the study of ion relaxation and transport mechanisms [9 and 10]. The shape of the loss tangent angle $\tan \delta(\omega)$ is actually sensitive to the imaginary part of the permittivity $\varepsilon_{\mathrm{r}}^{\prime \prime}(\omega)$ passes through a maximum at a frequency which is temperature dependent and whose inverse is commonly associated with the characteristic time required for dipoles to reorient.

In this contribution we present first results of combined acoustic and electrical investigation of $\mathrm{LiPO}_{3}$ metaphosphate

\footnotetext{
* ${ }^{1}$ Peter Hockicko, ${ }^{1}$ Peter Bury, ${ }^{2}$ Francisco Munoz, ${ }^{2}$ Laura Munoz-Senovilla

${ }^{1}$ Department of Physics, Faculty of Electrical Engineering, University of Zilina, Slovakia

${ }^{2}$ Instituto de Ceramica y Vidrio (CSIC), Madrid, Spain

E-mail:hockicko@fyzika.uniza.sk
} 
glasses. Double Power Law (DPL) model is used for description of experimental results obtained from acoustic spectra of the glasses with the purpose to study ion relaxation processes and transport mechanisms. Electrical measurements characterizing the ion relaxation processes and possible transport mechanisms in investigated ion conducting glasses are presented. The results obtained by different methods are then summarized and compared.

\section{Theoretical principles}

The acoustic attenuation will exhibit a maximum when the condition $\omega \tau$ is equal to 1 and

$$
\tau=\tau_{0} \exp \left(\frac{E_{a}}{k_{B} T_{\text {peak }}}\right)
$$

is the most probable relaxation time, $T$ thermodynamic temperature, $k_{B}$ the Boltzmann constant, $\omega=2 \pi v$ and $v$ is frequency of applied acoustic waves. The relaxation processes, described by an Arrhenius equation (1), are characterized by activation energy $E_{a}$ for jumps over the barrier between two potential minima and typical relaxation frequency of ion hopping $1 / \tau_{0} \approx 10^{13}-10^{14} \mathrm{~s}^{-1}$. An Arrhenius - type relaxation between the temperature of peak maximum $T_{\text {peak }}$ and the applied frequency $v$ of acoustic waves can be then expressed as

$$
v=v_{0} \exp \left(-\frac{E_{a}}{k_{B} T_{\text {peak }}}\right),
$$

where $v_{0}$ is the preexponential factor [11]

Double Power Law (DPL) function (3) has been mainly used to fit mechanical loss data in investigated glassy materials [11 and 12]

$$
\alpha(\omega, T) \propto \frac{1}{(\omega \tau)^{-n}+(\omega \tau)^{m}} .
$$

Using this function, we can fit also the acoustic attenuation spectrum of the lithium metaphosphate glass.

Determination of the imaginary part of the complex dielectric constant $\left(\varepsilon^{\prime \prime}\right)$ compared to the real part $\left(\varepsilon^{\prime}\right)$ play a crucial role in the study of fundamental properties of investigated materials [13], where $\varepsilon^{\prime}(\omega)$ and $\varepsilon^{\prime \prime}(\omega)$ characterize the refractive and absorptive properties of the material, respectively. The loss tangent defined by the relation

$$
\tan \delta(\omega)=\frac{\varepsilon^{\prime \prime}(\omega)}{\varepsilon^{\prime}(\omega)},
$$

can be also used for the relaxation spectroscopy that reflects the basic features of the relaxation processes of mobile ions. This quantity is related to the attenuation constant (or absorption coefficient) of an electromagnetic wave propagating in a material. The activation energies of the relaxation processes can be estimated from the plots of $\log f$ vs. $1 / T_{\text {max }}$ were $T_{\max }$ can be found using the isochronal peaks of $\tan \delta(\omega, T)$. These plots are straight lines in accordance with Arrhenius equation

$$
f=f_{0} \exp \left(-\frac{E_{a}^{t g \delta}}{k_{B} T_{\text {peak }}}\right),
$$

where $f$ is the frequency of the applied electrical field, $f_{0}$ is the pre-exponential factor. $E_{a}^{\operatorname{tg} \delta}$ is the activation energy associated to the dielectric loss [14].

The electrical conductivity $\sigma$ of many solids, including glasses, polymers and crystals, has been shown to consist of a frequency independent and a strongly frequency dependent component [15]. Experimental data in a limited frequency region revealed that the overall frequency dependence of $\sigma$ or the so called "universal dynamic response" (UDR) of ionic conductivity can be approximated by the following simple relation

$$
\sigma(\omega)=\sigma_{D C}+A \omega^{s}=\sigma_{0} \exp \left(-\frac{E_{a}^{D C}}{k_{B} T}\right)+A \omega^{s},
$$

where $\omega(=2 \pi f)$ is the angular frequency of measurement. Both $\sigma_{\mathrm{DC}}$ and $A$ follow Arrhenius type strong temperature dependencies. In Eq. $6 \sigma_{0}$ is the pre-exponential factor, $E_{a}^{D C}$ is the activation energy of the ion transport through hopping processes which can be determined from the DC measurements.

\section{Experimental}

A lithium metaphosphate glass with composition $50 \mathrm{Li}_{2} \mathrm{O} .50 \mathrm{P}_{2} \mathrm{O}_{5}$ was prepared by conventional melt-quenching procedure. Reagent grade raw materials analytically pure $\mathrm{Li}_{2} \mathrm{CO}_{3}$ and $\left(\mathrm{NH}_{4}\right)_{2} \mathrm{HPO}_{4}$ were mixed in stoichiometric amounts and the batch was calcined in a porcelain crucible up to $450{ }^{\circ} \mathrm{C}$, in an electric furnace, then melted during $2 \mathrm{~h}$ at $800{ }^{\circ} \mathrm{C}$. The melt was poured onto a brass mould and annealed slightly above its glass transition temperature, previously determined through Differential Thermal Analysis.

Experimental arrangement for acoustic attenuation measurement has been already described in [16]. The longitudinal acoustic waves of frequency $13 \mathrm{MHz}$ were generated by the Pulse Modulator and Receiver - MATEC 7700 and a $\mathrm{LiNbO}_{3}$ transducer acoustically bonded to a quartz rod buffer. The computer system was used to trigger the apparatus, to record the attenuation changes and to evaluate obtained acoustic spectra. We performed acoustic measurements of the signal going only through the rod buffer without studied sample. So that for the sample investigation we have used only acoustic attenuation on investigated sample. The electrical conductivity measurements were performed by the FLUKE PM 6306 RCL impedance analyzer at frequencies in the range $50 \mathrm{~Hz}$ to $1 \mathrm{MHz}$. The acoustic and electrical measurements 
were carried out at temperatures ranging from $290 \mathrm{~K}$ to $560 \mathrm{~K}$ (to $640 \mathrm{~K}$ for acoustic measurements) at a heating rate of $0.5 \mathrm{~K} / \mathrm{min}$.

The sample for acoustic and electrical measurements was square in shape (thickness $\approx 1.82 \mathrm{~mm}$ and area $=10.1 \times 10.1$ $\mathrm{mm}^{2}$ ) and end faces were polished to be flat and parallel.

\section{Experimental results and discussion}

The acoustic attenuation spectrum (Fig. 1) indicates one broad attenuation peak with a maximum at a temperature about $530 \mathrm{~K}$. Applying DPL function (3) we could simulate acoustic attenuation spectra at constant frequency as a superposition of individual peaks and determined the values of activation energies of the individual relaxation processes connected with ion hopping. The other peak occurring at the temperature higher than $590 \mathrm{~K}$ are connected with the processes appearance above the glass transition temperature, $T_{g}$ (for investigated sample $T_{g}=$ $587 \mathrm{~K}$ ).

The whole temperature dependence of acoustic attenuation has been analyzed assuming the existence of a thermally activated relaxation process associate to $\mathrm{Li}^{+}$ions. Using the theoretical double power law (DPL) model (Eq. 3), and Arrhenius equation (Eq. 2), we calculated the energy of the main relaxation process, $E_{a l}=0.73 \mathrm{eV}$ from acoustic attenuation measurement. This value is very close to the activation energy for conduction found from DC measurements $E_{a}^{D C}=0.735 \mathrm{eV}$ (Fig. 2). Using AC measurements the activation energy of the dielectric losses $E_{a}^{\operatorname{tg} \delta}=$ $0.79 \mathrm{eV}$ was obtained (Fig. 3).

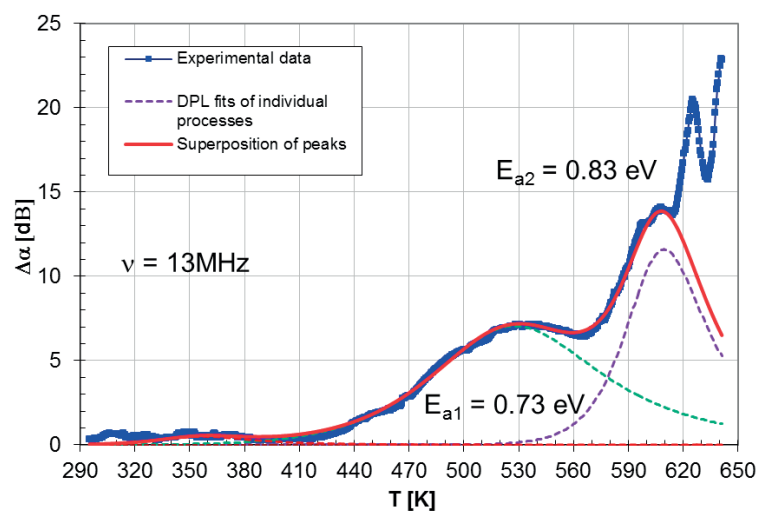

Fig 1. The acoustic attenuation spectra of investigated lithium metaphosphate glass measured at frequency $v=13 \mathrm{MHz}$ (full blue line) and the DPL fits of the supposed relaxation processes (dashed lines) including their superposition (full red line)
We have found that activation energy for conduction $E_{a}^{D C}$ is very close to activation energy $E_{a l}$ of the main relaxation process calculated from acoustic measurement which can be connected with the same microscopic process. However, the acoustic spectra detected also a second process, which could be related to the appearance of the glass transition with activation energy $E_{a 2}=0.83$ $\mathrm{eV}$. It is known that the conductivity of glasses shows a change in activation energy when passing over the glass transition [17]. Therefore, the second thermally activated process that has been seen through the acoustic measurements, with $E_{a 2}=0.83 \mathrm{eV}$, could correspond to movement of $\mathrm{Li}^{+}$ions above $T_{g}$

Complex impedance $\left(Z^{*}=Z^{\prime}-j Z^{\prime \prime}\right)$ plots at the temperatures from $T=330-390 \mathrm{~K}$ are illustrated in the insert of Fig. 2. The Nyquist plot consists of a depressed semicircle. The $\sigma_{D C}$ was calculated using the relation $\sigma_{D C}=d /(R A)$, where $d$ is the thickness of the bulk glass piece and $A$ is the area of cross section of the glass piece, resistance $R$ was calculated from the Nyquist plot. The DC conductivity was observed above $310 \mathrm{~K}$ and is ranged from $6.5 \times 10^{-9} \mathrm{~S} / \mathrm{cm}$ at $330 \mathrm{~K}$ to $2.1 \times 10^{-4} \mathrm{~S} / \mathrm{cm}$ at $550 \mathrm{~K}$. The increase of DC conductivity with the temperature indicates a thermallv activated conduction mechanism. The representative

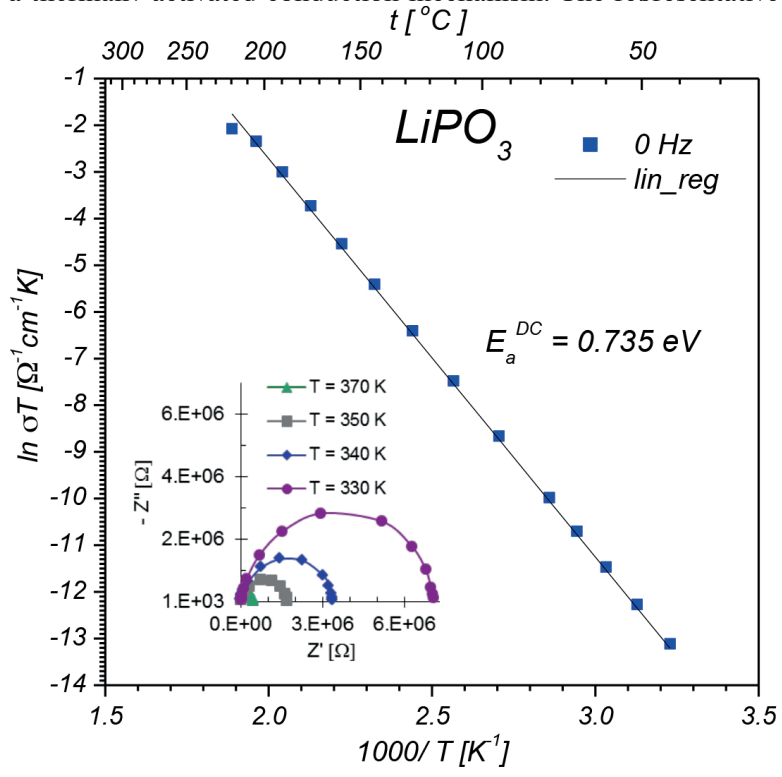

Arrhenius plot for investigated sample is illustrated in Fig. 2.

Fig. 2 Arrhenius plot constructed from temperature dependences of DC conductivity calculated from Nyquist diagram (inside)

Plotting the frequency of AC electric field in logarithmic scale as a function of corresponding inverse temperature of maximum of loss tangent angle (Arrhenius plot), the activation energy of the dielectric losses have been obtained. 


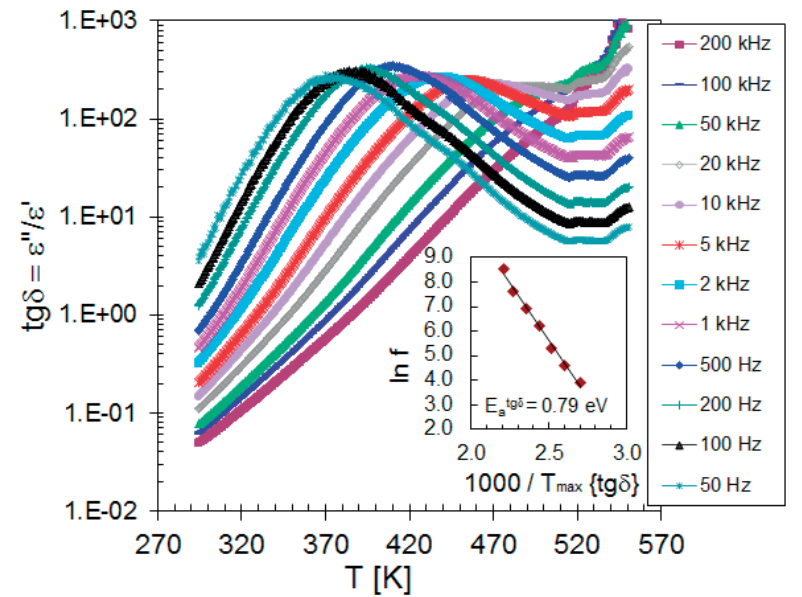

Fig. 3 Temperature dependence of loss tangent and Arrhenius plot of dielectric loss angle tangent maxima and applied frequency

The set of the frequency dependencies of AC conductivity measured at various temperatures (conductivity spectra) is illustrated for the glass in Fig. 4. The obtained AC conductivity measurements correspond to the complete conductivity spectra of glassy samples. However, because of the limited frequency range only two regimes (II and III) of the whole conductivity spectra represented by a different slope of individual curves could be recognized, moreover the regime II only at low temperatures. The low frequency part in regime III and the dispersive regime II are due to the hopping motion of the mobile ions. Adopting the Eq. 6 , slopes can be derived at the higher frequencies with parameter $s=0.68$ for temperature $310 \mathrm{~K}$ (Fig. 4 ).

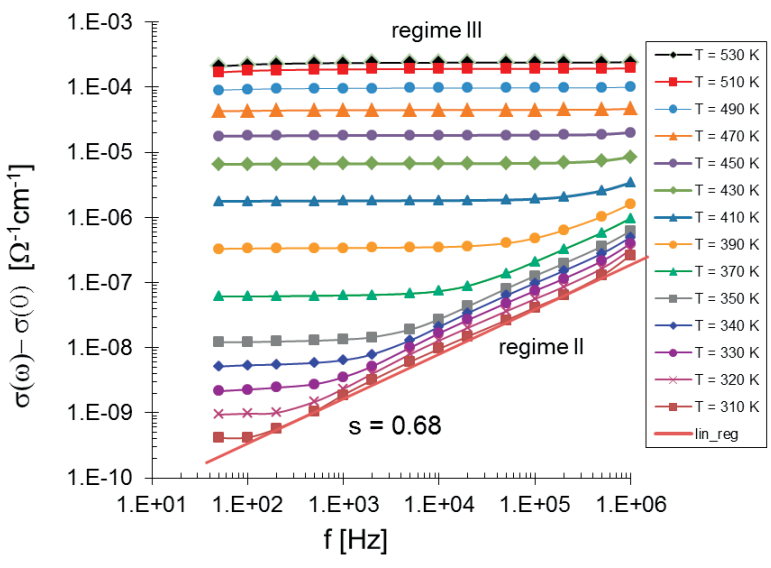

Fig. 4 Results obtained from AC electrical conductivity measurements

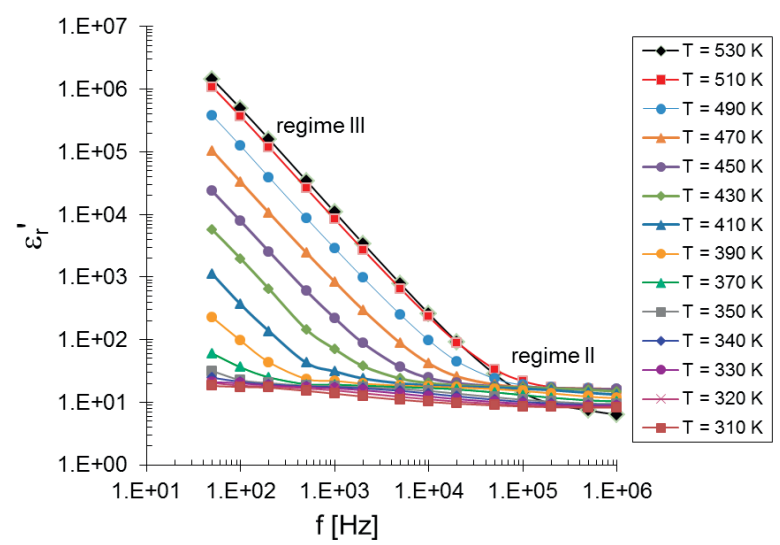

Fig. 5 Frequency dependences of the real part of complex relative permittivity

The real part of the complex relative permittivity versus frequency has been reported in Fig. 5. The behaviour of conductivity and permittivity is divided in two regimes: regime II can be observed only at low temperatures ( $310 \mathrm{~K}-350 \mathrm{~K})$. The conductivity at low temperatures and high frequencies is increased dramatically with the frequency and the permittivity is almost the same in anyway of the frequency and the temperature. Regime III is appeared at higher temperatures. The conductivity is almost constant and corresponds to the $\sigma_{\mathrm{DC}}$. The permittivity curve shows a slope. In this frequency range, ions jump from site to site through the bulk of the electrolyte. This corresponds to the random diffusion of $\mathrm{Li}$ ions in a disorder matrix [18]. In some materials [18 and 19] regime I can be observed: at low frequencies and high temperatures the ionic conductivity decreases drastically. The permittivity is very high and much constant for the lowest frequencies but decreases quickly when the frequency increases. This low-frequency part is influenced by the electrode polarization effect and the $\mathrm{Li}^{+}$ions are piled up on the blocking metal electrode.

\section{Conclusion}

Acoustical and conductivity measurements and dielectric relaxation behaviour have been examined over a wide temperature range in the glass with metaphosphate composition $\mathrm{LiPO}_{3}$. Using the theoretical model of Double Power Law function for the simulation of acoustic spectra we have determined the main relaxation process. Comparison the activation energies obtained from acoustic and electric DC measurements showed that the same microscopic phenomena can be responsible for the acoustic and electrical relaxation processes. 


\section{Acknowledgements}

The authors would like to thank to Mr. F. Cernobila for technical assistance. This study was supported by $R \& D$ operational program Centre of excellence of power electronics systems and materials for their components II. No. OPVaV2009/2.1/02-SORO, ITMS 26220120046 funded by European regional development fund (ERDF) and by project KEGA No. 035ZU-4/2012 and VEGA No. 1/0853/13. Funding from project MAT2010-20459 of MINECO (Spain) is acknowledged and L. Munoz-Senovilla also thanks the MINECO for her $\mathrm{PhD}$ scholarship (BES-2011-044130).

\section{References}

[1] DUCLOT, M., SOUQUET, J.-L.: J. Power Sources, 97-98, 2001, 610-615.

[2] BATES, J. B., DUDNEY, N. J., GRUZALSKI, G. R., ZUHR, R. A., CHOUDHURY, A., LUCK, C. F., ROBERTSON, J. D.: Solid State Ionics 53-56, 1992, 647-654.

[3] YU, X., BATES, J. B., JELLISON JR., G.E., HART, F. X.: J. Electrochem. Soc. 144 (2) 1997, 524.

[4] MUNOZ, F., DURAN, A., PASCUAL, L., MONTAGNE, L., REVEL, B., RODRIGUES, A. C. M.: Solid State Ionics 179 (15-16), 2008, 574-579.

[5] MASCARAQUE, N., FIERRO, J. L. G., DURAN, A., MUNOZ, F.: Solid State Ionics 233(0), 2013, 73-79.

[6] BROW, R. K.: Review: The Structure of Simple Phosphate Glasses, J. Non-Cryst. Solids 263\&264, 2000, 1-28.

[7] ROLING, B., HAPPE, A., INGRAM M. D., FUNKE, K.: J. Phys. Chem. B 103, 1999, 4122.

[8] FUNKE, K.: Sol. State Ionics 94, 1997, 27.

[9] ALMOND, D. P., WEST, A. R.: Solid State Ionics 26, 1988, 265.

[10] KUDELCIK, J., BURY, P., ZAVISOVA, V., TIMKO, M., KOPCANSKY, P.: Communications - Scientific Letters of the University of Zilina 12 (2), 2010, 34-37.

[11] ROLING, B., INGRAM, M. D.: Physical Review B 57 (22), 1998, 14192 - 14199.

[12] BURY, P., HOCKICKO, P., JAMNICKY, M.: Advanced Materials Research 39-40, 2008, 111.

[13] VENKATESH, J., SIVASUBRAMANIAN, V., SUBRAMANIAN, V., MURTHYA, V. R. K.: Materials Research Bulletin 35 (8), 2000, 1325-1332.

[14] PeVZNER, B., HeBARD, A. F., DRESSElHAUS, M. S.: Physical Review B 55 (24), 1997, 16439 - 16449.

[15] JONSCHER, A. K.: Nature 267, 1977, 673-679.

[16] HOCKICKO, P., BURY, P., MUNOZ, F.: J. Non-Crystalline Solids, 363 (1), 2013, 140-146.

[17] HALl, A. , SWENSON, J., BOWRON, D. T., ADAMS, S.: J. Phys.: Condens. Matter 21, 2009, 245106 (7pp).

[18] LE VAN-JODIN, L., DUCROQUET, F., SABARY, F., CHEVALIER, I.: Solid State Ionics 253, 2013, 151-156.

[19] HOCKICKO, P., BURY, P., MUNOZ, F.: Communications - Scientific Letters of the University of Zilina 15 (2a), 2013, 33-39. 